\title{
Cooperation Policies for Inter-organizational Workflows
}

Technical University of Vienna Information Systems Institute Distributed Systems Group

\author{
Issam Chebbi, Samir Tata and \\ Schahram Dustdar \\ Issam.Chebbi@int-evry.fr \\ Samir.Tata@int-evry.fr \\ dustdar@infosys.tuwien.ac.at
}

TUV-1841-2004-20

October 27, 2004

\begin{abstract}
In the context of inter-organizational workflows, interactions between partners should be constructive, complementary, and guide the work toward the objective of organizations involved in the workflow. Within our approach, inter-organizational workflow cooperation, which consists of workflow advertisement, workflow interconnection and workflow cooperation, this paper focuses on these cooperation policies one has to define in order to enforce and control interactions between partners' workflows. The cooperation policies integrate the partners' roles as well as their coordination, the dataflows allowed to pass from one partner's workflow to another, and their intervisibility levels. The level of the visibility is used to preserve the partner privacy and know-how and is reduced as tiny as cooperation needs.
\end{abstract}

Keywords: cooperation policies, inter-organizational workflows, cooperative workflows 


\section{Cooperation Policies for Inter-organizational Workflows}

\author{
Issam Chebbi \\ GET/INT \\ 9, Rue Charles Fourier \\ 91011 Evry, France \\ Issam.Chebbi@int-evry.fr
}

\author{
Samir Tata \\ GET/INT \\ 9, Rue Charles Fourier \\ 91011 Evry, France \\ Samir.Tata@int-evry.fr
}

\author{
Schahram Dustdar \\ Vienna University of Technology \\ Argentinierstrasse 8/184-1 \\ A-1040 Wien, Austria \\ dustdar@infosys.tuwien.ac.at
}

\begin{abstract}
In the context of inter-organizational workflows, interactions between partners should be constructive, complementary, and guide the work toward the objective of organizations involved in the workfow. Within our approach, inter-organizational workflow cooperation, which consists of workflow advertisement, workflow interconnection and workflow cooperation, this paper focuses on these cooperation policies one has to define in order to enforce and control interactions between partners' workflows. The cooperation policies integrate the partners' roles as well as their coordination, the dataflows allowed to pass from one partner's workflow to another, and their intervisibility levels. The level of the visibility is used to preserve the partner privacy and know-how and is reduced as tiny as cooperation needs.
\end{abstract}

\section{Introduction}

Cooperation between partners within a virtual organization is established according to the needs for business and criteria of competence. This gives the cooperation a dynamic character, where partners are in constant evolution and could join or leave the virtual organization.

To have relevant and constructive interactions that complement each other and guide the work toward the objective of the virtual organization, we propose to describe the partners' interactions in terms of cooperation policies that integrate the partners' roles, the dataflow exchanged between partners, and their workflow views that will be exposed and accessed by external organizations.

This paper is organized as follows. Section 2 presents related work in the area of inter-organizational workflows. In Section 3, we summarize the three steps approach for interorganizational workflow cooperation we proposed in [4]. In Section 4, we propose a cooperation policy establishment process. Next, Section 4.2.1 presents an illustrative example. Conclusions and future work are presented in Section 5.

\section{Related Work}

For a number of years research on workflow management has focused on inter-organizational issues and many efforts have been done in this field.

In [5], the author presents many forms of workflows interoperability and focuses on the capacity sharing, chained execution, subcontracting, extended case transfer, loosely coupled, and public-to-private approaches. Problems to be encountered with those approaches are mainly autonomy of local workflow processing, confidentiality that prevents complete view of local workflow, and especially flexibility that needs no definition of global workflow that define cooperation between local workflows.

The inter-organizational cooperation problem has also been addressed by using the notion of agreements and contracts to define the business relationships between organizations. As an example, we can cite the CrossFlow approach [2]. However, this approach doesn't deal with arbitrary public processes and no standard definition language and semantics is provided for the enforcement of contracts between two organizations. In addition all involved organizations are required to use the same software for contract enforcement.

In [3], the authors present an approach for process management and coordination based on synchronization points between process services. This approach needs more flexibility in order to allow partners to personalize their internal processes without affecting the cooperation.

Virtual organizations are also addressed by the BPEL project that provides an XML notation for specifying business process behavior based on Web Services. However, $B P E L$ doesn't support the concepts that are paramount for the inter-organizational cooperation such as the notion of manual activities, applications, collaborative partners' heterogeneity... Moreover, the cooperation description that consists of linking roles to ports is limited and make it difficult to clearly express the collaboration. between two or more partners. 
In consequence, the absence of end-to-end process control, which is often related to the absence and/or limits of a single overall process ownership, has led workflow research to reexamine and to find new ways for workflow composition. One of the basic obstacles is the lack of a comprehensive approach to inter-organizational workflows as a basis for contracting and standardization.

\section{Approach to Workflow Cooperation}

In this section, we present the approach to interorganizational workflow cooperation we proposed in [4]. This approach consists of three steps: workflow advertisement, workflow interconnection and workflow cooperation. In the following we present these steps. Section 4 focuses on the second step of this approach.

Step 1: Workflow Advertisement Each organization has to advertise the semantic description of its offered and required activities. Partners' identification with complementary competencies and knowledge is based on an automated research process that is based on the wished cooperation level. The published information can be managed within an accessible registry that provides organizations with searching and publication capabilities, to share workflow semantic information, and to enable trading partners' identification.

Step 2: Workflow Interconnection Identified partners negotiate their roles within the virtual organization as well as the coordination of their workflows. The result of this step is a set of cooperation policies that describe especially the responsibilities and the roles played by the partners in the cooperation. For each organization, cooperation policies define the visibility levels of its workflows for its partners.

Step 3: Workflow Cooperation The last step consists in the monitoring as well as the control of cooperation between workflows with respect to the established cooperation policies.

\section{Cooperation Policy Establishment Process}

In this section, we suppose that the first step of our approach to inter-organizational workflow cooperation we have presented in section 3 is done. In fact, we consider here that partners within a virtual organization have identified each other and each requested service of any of their workflows can be fulfilled by an activity or a sub process of one other among their workflows. In this section, we present the notion of cooperation policies. We propose to define how workflows interact within a virtual organization and present a process for cooperation policy establishment.

\subsection{Cooperation Policy}

Cooperation policies describe (without explicit specification) a set of accepted interaction scenarios rather than one (which is the case of existing approaches since they use a workflow to specify interactions between workflows, see [6] for example). To do so, cooperation policies are defined between public view workflows rather than the workflows themselves. This can lead the different partners in a virtual organization to preserve their know-how using the notion of views. A public view workflow is composed by virtual activities and the control flow between them. A virtual activity can be connected to one or several activities belonging to one workflow and represents the level of the visibility used to preserve privacy and its know-how as well as to allow interactions with cooperating partners. The intervisibility is reduced as tiny as cooperation needs [1].

Connections between virtual activities and "real" ones can be changed without changing the coordination (or flow control) between virtual activities. This can allow to each organization to adapt and/or change its internal workflow structure without changing its role in the cooperation.

Before introducing cooperation policies process establishment that define the public views of each workflow within a virtual organization, lets define the notion of a public view workflow as well as the notion of cooperative workflow.

\subsection{Cooperative and Public View Workflows}

4.2.1. Cooperative Workflows A cooperative workflow is composed of all the cooperative activities and their cooperative control flow.

Cooperative Activity For a participating workflow, we call a producing cooperative activity, any activity that produces a dataflow for an external activity that belongs to another workflow. We call a consuming cooperative activity, each activity that consumes a dataflow from an external activity that belongs to another workflow. A cooperative activity can be a producing and/or consuming one.

Cooperative Control Flows Figure 1 recapitulates all the relevant control flows that must be preserved when passing from the internal workflows to the cooperative ones. A non-cooperative control flow is a control flow that does not participate directly in the cooperation with other workflow.

In figure 1, we show that cooperative control flows are: control flows starting from and ending in a cooperative activity, control flows starting from a producing cooperative activity and ending in an external activity, or control flows starting from an external activity and ending in a consuming cooperative activity.

Example To illustrate the notions we propose here, we give, in the following, an example (inspired by [5]) that involves 


\begin{tabular}{|c||c|c|c|c|}
\hline \multicolumn{1}{|c|}{ Control flow } & $\begin{array}{c}\text { Internal } \\
\text { activity }\end{array}$ & $\begin{array}{c}\text { Producing } \\
\text { cooperative } \\
\text { activity }\end{array}$ & $\begin{array}{c}\text { Consuming } \\
\text { coperative } \\
\text { activity }\end{array}$ & $\begin{array}{c}\text { External } \\
\text { activity }\end{array}$ \\
\hline \hline Internal activity & non-cooperative & non-cooperative & non-cooperative & irrelevant \\
\hline $\begin{array}{c}\text { Producing } \\
\text { cooperative activity }\end{array}$ & non-cooperative & cooperative & cooperative & cooperative \\
\hline $\begin{array}{c}\text { Consuming } \\
\text { cooperative activity }\end{array}$ & non-cooperative & cooperative & cooperative & irrelevant \\
\hline External activity & irrelevant & irrelevant & cooperative & irrelevant \\
\hline
\end{tabular}

Figure 1. Cooperative Control Flow

a virtual organization composed of four business partners: a customer, a producer and two suppliers. We suppose here that these partners have identified each other and each requested service of any of their workflows can be fulfilled by an activity or a sub process of the virtual organization.

In this example, a customer initiates the cooperation by sending an order to the producer who orders two needed products, $b$ and $c$, assembles them and delivers them to the consumer. Figure 2 illustrates the four partners' workflows.

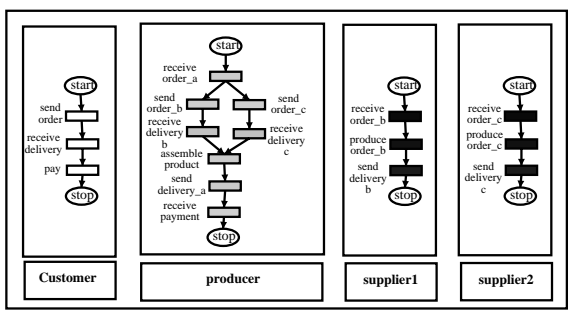

Figure 2. Partners' workflows

Figure 3 presents the cooperative workflows of the four partners composed of only cooperative activities and control flows (see rules of figure 1).

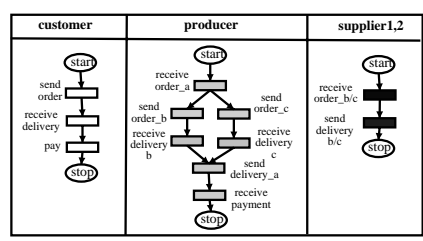

Figure 3. Partners' Cooperative Workflows

4.2.2. Public View Workflows A public view workflow is created when a workflow wants to expose some activities or to access activities of workflows of other organizations. A public view workflow is the public view of the cooperative workflow. It consists of virtual activities, which represent a subset of the cooperative workflow activities.
Virtual activities are not supposed to produce or consume output/input. Nevertheless, they are supposed to transfer output/input data to/from other a workflow. Similarly, they are not meant to be executed by a local role.

To define the public view workflows to an interorganizational workflow, and roles and responsibilities of the different partners within a virtual organization, we propose in the following section a process for cooperation policy establishment.

\subsection{Cooperation Policy Establishment Process}

4.3.1. Establishment Process In order to establish a cooperation policy, we propose a three phases process: An access contract establishment to express allowed activities one partner can execute on its behalf by an external partner, a dataflow contract establishment to express dataflows to be exchanged between partners and workflows public views definition.

Phase 1 : Access Contract Establishment The objective of this phase is to determine, for each partner, the set of external activities (activities executed by external workflows on its behalf) it can execute as well their coordination. This phase consists of identifying the roles of the different identified partners, as well as the coordination of their cooperative activities. Hence, the result of this phase is a set of rules describing the responsibilities and the roles played by each partner in the cooperation. We call this set of rules an access contract.

Phase 2 : Dataflow Contract Establishment After determining the different activities participants' workflow can be executed on its behalf within an external workflow, each partner specifies the different dataflows to exchange with the other partners. The result of this step is a set of rules associating, in a peer to peer manner, the partners with the data they can send to each other. We call this set of rules a dataflow contract.

Phase 3 : Public Views Definition Based on the access and dataflow contracts, each partner defines the visibility levels of its workflow to the other partners. Hence, many views permitting to cooperate without revealing its know-how are generated. This allows providing each partner with only information it needs to know by concealing all details and extra-information. This reduces the inter-visibility as tiny as the cooperation needs.

4.3.2. Example The next step of the example presented in section 4.2.1 consists of defining, for each partner, all the activities that could be executed on its behalf by external organizations as well as their coordination.

Phase 1 : Access Contract Establishment In figure 4, we give the three access contracts between the four partners. 


\begin{tabular}{|l|c|c|}
\hline \multicolumn{3}{|c|}{ Customer-Producer Access Contract } \\
\hline \multicolumn{1}{|c|}{ Customer } & Producer & Order \\
\hline * Receive_Order & * Receive_Delivery & * Receive_Order \\
* Receive_Payment & & $\begin{array}{l}\text { * Receive_Delivery } \\
\text { * Receive_Payment }\end{array}$ \\
\hline
\end{tabular}

Producer-Supplier $1 / 2$ Acces Contract

\begin{tabular}{|c|c|c|}
\hline Producer & Supplier/2 & Order \\
\hline * Receive_Order $/ \mathrm{c}$ & * Receive_Delivery $/ \mathrm{c}$ & $\begin{array}{l}{ }^{*} \text { Receive_Ordet } / \mathrm{c} \\
{ }^{*} \text { Receive_Delivery }\end{array}$ \\
\hline
\end{tabular}

Figure 4. Partners Access Contracts

Phase 2 : Dataflow Contract Establishment After the access contract establishment, each partner specifies the dataflows to be exchanged with the partners. The result of this step is illustrated in figure 5

\begin{tabular}{|c|c|c|c|}
\hline \multicolumn{2}{|c|}{ Customer-Producer Dataflow Contract } & \multicolumn{2}{|c|}{ Producer-Supplier 1/2 Dataflow Contrac } \\
\hline Customer & Producer & \begin{tabular}{|l|} 
Producer \\
\end{tabular} & Supplien/2 \\
\hline $\begin{array}{l}* \text { order_a } \\
* \text { payment }\end{array}$ & $\begin{array}{l}\text { * delivery_a } \\
* \text { ionvoice }\end{array}$ & $* \operatorname{Order}_{\mathrm{b} / \mathrm{c}}$ & ${ }^{*}$ Delivery $/ \mathrm{c}$ \\
\hline
\end{tabular}

Figure 5. Partners Dataflow Contracts

Phase 3 : Public Views Definition Based on the previous access and dataflow contracts, each partner generates one or more views permitting to cooperate without revealing its know-how on the one hand and allowing to provide each partner with only information it needs to know on the other hand. Hence, the customer provides only one workflow view composed of two virtual activities and permitting to cooperate with the producer. The producer generates three workflow views. The first is composed of three virtual activities and allows the cooperation with the customer; the two others, each one is composed of two activities and permits to cooperate with one supplier. Finally, each supplier provides one workflow view to cooperate with the producer. Figure 6 shows the partners' public view workflows.

\begin{tabular}{|c|c|c|c|c|}
\hline customer & \multicolumn{3}{|c|}{ producer } & supplier1,2 \\
\hline Producer View & Customer View & Fupplier1 Viev & Supplier2 View & Producer View \\
\hline صת & קי & & & \\
\hline 宁 & & t & 2 & \\
\hline
\end{tabular}

Figure 6. Partners' Public Views

After contracts establishment and the public views generation, the different partners are ready to interconnect and cooperate. Figure 7 shows the partners' interconnection via their public views.

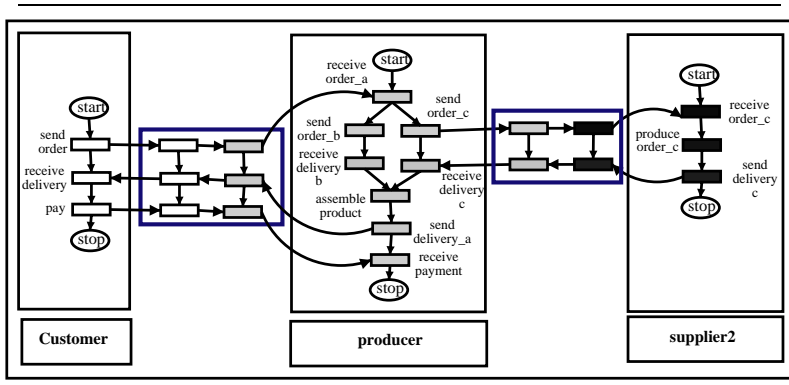

Figure 7. Partners' Interconnection

\section{Conclusion and Future Work}

A cooperation policy establishment process was presented in this paper within a flexible approach. Indeed, it allows dynamic interactions between different organizations composing a virtual organization and the concept of public views permit the cooperative workflows internal structures adaptation without changing the inter-organizational workflows and varying degrees of workflows visibility which enables organizations to retain required levels of privacy and security of internal workflows.

Finally, our ingoing and future work includes a prototype system development permitting a dynamic join and disconnection of partners, monitoring and control of interaction according to established cooperation policies.

\section{References}

[1] K. Baina, S. Tata, and K. Benali. A model for process service interaction. In W. van der Aalst, A. ter Hofstede, and M. Weske, editors, Business Process Management: International Conference, LNCS 2678, Eindhoven, The Netherlands, June 26-27 2003. Springer-Verlag Heidelberg.

[2] P. Grefen, K. Aberer, Y. Hoffner, and H. Ludwig. Crossflow: Cross-organizational workflow management in dynamic virtual enterprises. International Journal of Computer Systems Science \& Engineering, 15(5):277-290, 2000.

[3] O. Perrin and C. Godart. A model to support collaborative work in virtual enterprises. Data \& Knowledge Engineering, 50(1):63-86, 2004.

[4] S. Tata and I. Chebbi. A bottom-up approach to interenterprise business processes. In Distributed and Mobile Collaboration, 13th IEEE International Workshops on Enabling Technologies, Modena Italy, June 2004.

[5] W. M. P. Van Der Aalst. Process-oriented architectures for electronic commerce and interorganizational workflow. Inf. Syst., 24(9):639-671, 1999.

[6] W. M. P. van der Aalst and M. Weske. The $\mathrm{p} 2 \mathrm{p}$ approach to interorganizational workflows. In Proceedings of the 13th International Conference on Advanced Information Systems Engineering, pages 140-156. Springer-Verlag, 2001. 